\title{
„Papiere welche die Nachwelt nicht zu beschnobern braucht". Gottfried August Bürger als Briefschreiber
}

"Manuscrits dont la postérité pourra se passer ».Gottfried August Bürger épistolier

"Manuscripts that posterity will bypass". Gottfried August Burger, a letter writer

Ulrich Joost

\section{(2) OpenEdition}

\section{Journals}

Édition électronique

URL : http://journals.openedition.org/ceg/970

DOI : $10.4000 /$ ceg. 970

ISSN : 2605-8359

\section{Éditeur}

Presses Universitaires de Provence

\section{Édition imprimée}

Date de publication : 30 mai 2016

Pagination : 193-210

ISBN : 979-10-320-0067-0

ISSN : 0751-4239

\section{Référence électronique}

Ulrich Joost, « „Papiere welche die Nachwelt nicht zu beschnobern braucht". Gottfried August Bürger als Briefschreiber », Cahiers d'Études Germaniques [Online], 70 | 2016, Online erschienen am: 17 Dezember 2017, abgerufen am 26 November 2020. URL : http://journals.openedition.org/ceg/970 DOI : https://doi.org/10.4000/ceg.970 


\section{„Papiere welche die Nachwelt nicht zu beschnobern braucht“ \\ Gottfried August Bürger als Briefschreiber}

Ulrich JOOST

Technische Universität Darmstadt

\section{Vernichten oder Bewahren - das Profil eines wichtigen Textcorpus'}

Der Titel meines Referats ${ }^{1}$ zitiert eine Selbstanweisung Bürgers, von der er einem Korrespondenten berichtet:

Hast Du noch so viel Zeit und Kräfte, dachte ich, so willst du von deinen Papieren welche die Nachwelt nicht zu beschnobern braucht, verbrennen lassen, was [=wes] du habhaft werden kannst, oder dein Freund Althof soll den ganzen Wust einstweilen zusammenraffen, und nach deinem Tode thun, was du nicht mehr thun konntest. (an Oesfeld, 14. 3. 1794) ${ }^{2}$

Aber auch schon sechzehn Jahre früher (und noch nicht resigniert) war Bürger sich der Brisanz und Peinlichkeit seiner Briefe wohl bewusst. Er fasste bereits - im Unterschied zu vielen seiner Zeitgenossen wie den Korrespondentenzirkeln um Gleim oder Lavater - die Privatheit des Briefs und den Zirkel der Empfänger sehr viel enger. Seinem Verleger Johann Christian Dieterich etwa, befiehlt er am 23. 4.1778 - und das ist eine unter mehreren Anweisungen von dieser Art:

1. Meine in Toulouse mit Rücksicht auf die Redezeit aufs Äußerste verknappt und nur ausgewählt vorgetragenen Überlegungen können jetzt etwas vollständiger mitgeteilt werden; der Vortragston wurde aber weitgehend erhalten. Die Abhandlung will einige Grundlinien und -fragen von Bürgers Briefstil in ihrem kultur-, stil- und ästhetikgeschichtlichen Kontext erörtern, beansprucht aber, weder vollständig und abschließend, noch neuerungssüchtig in theoretischer oder methodischer Hinsicht zu sein. Ich beschreite ähnliche Wege wie in meiner umfangreicheren Untersuchung Lichtenberg - der Briefschreiber (Göttingen 1993), dessen Briefstil und Kommunikationsprogramm manche Parallelen zu Bürger aufweist (z. B. in Intertextualität, Empfängerzugewandtheit, rhetorischen Strategien und sprachliche Modernität), aber auch tiefgründige Unterschiede (ästhetische Innovation). Ganz übergangen habe ich hier Bürgers Programm der Bildlichkeit, das unbedingt einer eigenen Untersuchung gerade auch im Kontext des poetischen Werks wert wäre.

2. Bürgers Briefe zitiere ich im Folgenden nach dem Arbeitsmanuskript meiner noch in statu nascendi befındlichen Edition, deren erster von fünf geplanten Bänden in diesem Jahr erschienen ist: Ulrich Joost, Udo Wargenau u. a. (Hrsg.), Gottfried August Bürger: Briefwechsel. Kritische und kommentierte Gesamtausgabe in 5 Bänden, Bd. 1: 1760-1776, Göttingen, Wallstein, 2015. Die weiteren Bände sollen ungefähr im Jahresrhythmus folgen. 
Wenn Sie mich lieb haben, so verbrennen Sie diejenigen meiner Briefe, deren Inhalt nur für Sie allein war; und zu weiter nichts, als zum Lachen dienen solte.

Nicht minder deutlich heißt es an Heinrich Christian Boie unterm 12. 3. 1778:

so lege ja meine Briefe nicht weit von der Hand, sondern las ein Kohlfeüer neben dich stellen und wirf sie hinein. Ich würde schön gekreüziget werden, wenn über so manchen Stellen, wie der vorhergehenden, ein unberufener Herausgeber einmal kommen solte. Ich habe mit den Briefen meiner Freünde mir das nehmliche vorgenommen.

Glücklicherweise hat er selber diesen Plan nicht perfekt verwirklicht. Ein großes Autodafé, wie Goethe es 1770 und 1797 gleich zweimal veranstaltete und den größten Teil der Briefe an ihn und vermutlich dann auch Entwürfe eigener Schreiben vernichtete ${ }^{3}$ und damit ganz gewiss die eigene Jugendgeschichte für sich selber und vor allem für die Nachwelt weniger auszulöschen als der Deutungshoheit der eigenen retrospektiven autobiographischen Darstellungen zu unterwerfen suchte, fand durch Bürger oder seinen Kreis offenbar nicht statt. Und auch seine Korrespondenten, ob Freunde, Kollegen oder Widersacher, hielten sich anscheinend in den seltensten Fällen an seine ja vermutlich auch nicht so ganz brod-kafkaisch ernstgemeinte Anweisung. Boie, der sanfte und keusche Boie, dem wohl nie ein unpassendes Wort entfahren sein dürfte, antwortete jedenfalls auf die eben zitierte Anweisung:

Unsere Briefe beiderseits sind nicht für die Augen andrer. Sobald einer von uns sich gefährlich krank fühlt, müßen sie ins Feuer. Aber so lang ich das nicht fühle, kommen sie nicht von mir. Ich lese und blättere oft in deinen Briefen, und freue mich unsrer Freundschaft. (19. 3. 1778)

Die meisten andern Briefempfänger dachten wohl ähnlich, und so hält sich vielleicht der (immer noch gewiss enorme) Schwund der Briefe Bürgers im üblichen Rahmen der Zufallsverluste infolge von Umzügen, Aufräumen und Erbfolgen. Und am Ende war sich Bürger seines Wertes überaus und immer bewusst; solche Zeilen wie die folgenden an Philippine Gatterer sind keine bloße Koketterie oder gar Ironie:

Vor allen Dingen lassen Sie sich gesagt seyn, daß Sie alle Ihre Episteln Briefe und Sendschreiben in einerley Format, wie ich auch thun werde, schreiben müssen, damit man sie desto bequemer in das Archiv legen und hernach ohne viele Umstände in die Druckerey schicken kann. (10. 9. 1777)

Leider haben die späteren Nachlassverwalter und vor allem auch seine Herausgeber nachträglich dann doch die vorgeblich gewünschte Vernichtung betrieben. Jedenfalls ist die Geschichte der Edition von Bürgers Korrespondenz (im Unterschied zu seinem poetischen Werk) alles andere als ein Ruhmesblatt der germanistischen Philologie; ist vielmehr ein Musterbeispiel an Verfälschung,

3. Das erste „Autodafé“ von Briefen (zuvor hatte er aber schon 1767 in Leipzig seine frühen Dichtungen verbrannt), fand 1770 statt; darüber berichtet er selbst sehr plastisch im 8. Buch von Dichtung und Wahrheit. Das zweite folgte dann im Sommer 1797 anlässlich der Italienreise (Tagebuch vom 9. 7. 1797; vgl. die entsprechenden Bemerkungen in den Tages- und Jahresheften). 
Unterdrückung, Unzulänglichkeit und Dilettantismus. Doch das soll uns hier und heute nicht interessieren: In ein paar Wochen erscheint der erste Band einer auf 5 Bände berechneten Edition des Briefwechsels, die alles noch Überlieferte zusammenfassen soll und von mir im Verein mit ein paar Kollegen bearbeitet wird; dort findet sich ein knapper Abriss der Editionsgeschichte. Soviel muss jetzt zur Kenntnis genommen werden, dass sich die Zahl der bei Erscheinen der ersten Gesamtausgabe, 1874 von Adolf Strodtmann in vier Bänden herausgegeben, noch vorhandenen knapp 900 Briefe annähernd verdoppelt hat. Ein Fünftel davon freilich sind juristische Schriftstücke, Briefe, Anweisungen, Protokolle, kurz: Aktenstücke aus der Amtmannstätigkeit, bei denen der Verlust durch die Aktenvernichtung des 19. Jahrhunderts und die Zerstörung Hannovers im Zweiten Weltkrieg, wo das Familienarchiv der Dienstherren Bürgers gerade angekommen war, noch erheblich größer war als bei den Privatbriefen. Aber selbst diese amtlichen oder halbamtlichen Briefschaften nicht mitgerechnet, gehört Bürger zu den stärkeren und besser überlieferten Briefschreibern seiner an Briefen doch unerhört fruchtbaren Zeit. ${ }^{4}$

Indes: Bürgers Forderung, die Briefe heiklen Inhalts zu vernichten, ist weit über das Philologische und über eine persönliche Koketterie hinaus von größtem Belang: Sein Postulat markiert ein Bewusstsein für die epochalen Wechsel wie - natürlich auch - seine permanenten Verstöße gegen Aptum und Decorum. Es markiert auch - das ist offenbar nie beachtet worden, wie sich sein Dichtungskonzept und dessen großes, durch Schillers Angriff besiegeltes Scheitern auch in seinem Briefstil programmatisch und textsortenspezifisch leicht erklärlich noch greller als in den öffentlichen Schriften abbildet. Denn größer als Bürgers andere Leistungen: die Kunstballade wiederbelebt, das Sonett erneut etabliert und mit den Münchhausen-Erzählungen ein wahres Volksbuch geschaffen zu haben (insofern außerhalb der akademischen Literaturforschung niemand mehr weiß, wer der Verfasser dieser Schwänke gewesen ist), dürfte meines Erachtens sein Entwurf einer sozusagen naturalistischen Ästhetik aus der Volkssprache gewesen sein, den Schiller ihm gründlich durch seine anonyme, nachher so berühmte gewordene Rezension in der Jenaer Allgemeinen

4. Unsere Gesamtausgabe fasst dabei den Brief-Begriff im Sinne des 18. Jahrhunderts weit, wobei der gesamte amtliche Briefverkehr in einem Sonderband mit eigener Chronologie ausgegliedert ist, wenn auch nach der gegenwärtigen Planung. (Das lässt gut zu begründen, insofern die dafür vorgesehenen Texte in einem gänzlich anderen Textsortenstil gehalten sind als die Freundes- und Kollegenkorrespondenz.) Es gehören aber fraglos hinein: die Korrespondenzen um die Ehescheidung, insofern dies ursprünglich privateste Rechtfertigungsschreiben waren; die Akten eines poetischen Wettstreits als Ausdruck einer untergegangenen Geselligkeitskultur wie überhaupt die poetischen Episteln, ob gedruckt, ob unveröffentlicht; möglicherweise die gedruckten Avertissements und die „Sogenannten Briefe“ (also Essays in Briefform ein im 18. Jahrhundert nicht erst, seit Lessing sie so getauft hat, ein überaus beliebtes literarisches Genre). Schließlich werden noch an sich einer andersartigen Kommunikationsstruktur gehorchenden Stammbuchblätter und mit noch größerer Berechtigung die Stellvertreterbriefe (wie die von Bürgers Frau an Goeckingk) sowie einige randständige Dokumente in der Chronologie eingeordnet. Purismus verbietet sich übrigens schon deswegen, weil dies mit Bestimmtheit die letzte Gelegenheit zu unseren Lebzeiten sein wird, derlei Dokumente aus Bürgers Leben und Wirken zwischen zwei Buchdeckel zusammenzufassen. 
Literaturzeitung verwies. Freilich prallten hier zwei anthropologisch unvereinbare Gegensätze aufeinander: auf der einen Seite der idealistisch-aufklärerische Anspruch, durch ästhetische Erziehung das ganze „Menschengeschlecht“ verbessern zu können, auf der anderen die Vorstellung, dass die reine ,Natur' nur durch Rückkehr zu den Wurzeln einer aus dem anonymen Volk entstammenden Sprache und Dichtung wieder erreicht werden könnte. Vielleicht war Bürger da durch Herders Geschichtsdenken und Ideen zur Poesie beeinflusst, ging aber sogar über die Ziele des Sturm und Drangs beträchtlich hinaus und nahm nebenbei Dichtungsvorstellungen der Jenaer Romantik vorweg. Wer in diesem Concurs indessen gewonnen hat und damit zugleich die Verwirklichung von Bürgers Konzept um ein volles Jahrhundert aufschob, wissen wir.

\section{Frühe Wirkung}

\section{Bürgers Korrespondenz in der Wahrnehmung seiner unmittelbaren Nachwelt}

Zuvörderst interessierte das Publikum an Bürgers Briefen das oft nur sensationell gesucht, zumal manchmal zutiefst unerfreuliche Biographische - wie ja bis heute und bei den meisten Briefeditionen, die wir selten wegen langweiliger Alltagsinformationen lesen wollen. Schon 1795 erschien im Genius der Zeit, einer Zeitschrift August von Hennings, jener Brief an Oesfeld, aus dem ich eingangs zitierte. ${ }^{5}$ Der gewisse haut gout der dritten Ehe mit nachfolgender Scheidung rief gleich mehrere Publikationen auf den Plan, und vor allem wurde der berühmte Rechtfertigungsbrief an die Schwiegermutter alsbald (1814 - und nicht einmal um die allerdings fürchterlich derb-direkten Stellen gekürzt) ${ }^{6}$ als „Bürgers Ehestandsgeschichte“ publiziert. Was für ein Missverständnis der Rezeption bis heute! Denn diesen Brief hat der Jurist Bürger doch wohl keineswegs geschrieben, um seine Frau zu verdammen, sich zu rächen oder auch nur sein Verhalten zu entschuldigen - nein, er brauchte den körperlichen Beweis eines zutiefst ehrlich wirkenden Protokolls, um sich von einer als vollkommen schuldig dastehenden Ehefrau scheiden zulassen, denn andernfalls hätte die zurückzuerstattende Mitgift ihn vollständig ruiniert.

Dass Bürger ein Briefschreiber par excellence gewesen ist, haben seine Zeitgenossen aber doch schon gewusst und die Nachkommen dementsprechend gebührend gewürdigt. Sein „körniger Stil“ hat wohl verhindert, dass er frühzeitig in die Liste der nachahmenswerten Briefschreiber im Konversations-Lexikon des 19. Jahrhunderts aufgenommen und in eine Reihe gestellt wurde mit Gellert, Lessing, Forster, Lichtenberg und Goethe, in die er gehört hätte. Aber gelesen

5. Genius der Zeit, 1795, 5. Stück, S. $41 \mathrm{ff}$.

6. Diese Kürzung nahm erst Strodtmann in seiner Ausgabe vor (4, 1874, S. 142-193). Ibid. S. 142 heißt es entschuldigend: „Die Weglassung einzelner, allzu widerwärtiger Details dieses Briefes ist durch . . . . bezeichnet.“ 
wurde er gern, das zeigen zahlreiche frühe Abdrucke seiner Briefe in den Zeitungen und Zeitschriften des Jahrhunderts. ${ }^{7}$

\section{literaturgeschichtlich, biographisch, bibliographisch}

Bürgers Korrespondenz ist selbst in den vollkommen unzulänglichen Editionen immer wieder herangezogen worden - und muss auch herangezogen werden. Er ist der einzige Zeitgenosse des Hains, von dem in dieser Korrespondenz ein bisschen mehr und zusammenhängend Informationen zum Literaturbetrieb um die Musenalmanache zu entnehmen ist; selbst die Voß-Korrespondenz ist nicht so reichhaltig und vor allem nicht so gut überliefert, und nur die Briefe von und an Heinrich Christian Boie, die bei weitem nicht vollzählig ediert, vielmehr nur ganz verstreut und unübersichtlich publiziert sind, nicht einmal bibliographisch ausreichend erfasst sind, übertreffen die Überlieferung bei Bürger. Bürger ist einer der ganz wenigen Beispiele von poetischen Herausgebern, die eben aus dieser beruflichen Tätigkeit heraus Kladden der eigenen Briefe wenigstens zu Teilen hinterließen, und diese Nachlassbestände waren die Basis von Strodtmanns Ausgabe. Wenige Quellen geben selbst nach den zahllosen willkürlichen Streichungen durch Strodtmann und andere Herausgeber so viel her für die Erkenntnis eines Schriftstellers, der versucht, von seiner Feder wenn nicht zu leben, so doch seine Existenz zu verbessern.

\section{kulturgeschichtlich}

Den Kulturhistoriker interessieren aber inzwischen und zu Recht noch ganz andere Fragen. Im großen Prozess der europäischen Briefgeschichte in der frühen Neuzeit steht Bürger tatsächlich an einer wichtigen Epochenschwelle, ist einer der Repräsentanten einer außerordentlichen Briefkultur, die wir mit den Namen solcher Dichter, Schriftsteller und Literaten verbinden wie Klopstock, Lessing, Lichtenberg, Boie, Goethe, Forster und Schiller, solcher Frauen wie Friederike Baldinger (ein bis heute nicht gehobener Schatz), Therese Huber, Caroline Michaelis und der anderen Romantikerfrauen. Hinzugefügt sei, dass wir Heutigen gerade das Ende jenes Zeitalters einer europäischen Briefkultur erleben, welches freilich schon Theodor Wiesengrund Adorno vor einem halben Jahrhundert anlässlich der Neuausgabe von Walter Benjamins „Deutschen Menschen“ für tot erklärt hatte - vermutlich war das bei Adorno ausgelöst durch den Schock des untergehenden und in der Barbarei versunkenen alten Europa. Uns wiederum Nachgeborenen verschwimmt die Epochengrenze des

7. Einen beileibe nicht vollständigen Überblick muss man sich durch Strodtmanns ziemlich gewissenhafte Quellenangaben verschaffen. Goedekes Grundriß 4 (3. Aufl. Abtlg., Dresden, Ehlermann, 1916, S. 993-996) bietet bibliographisch aufs Äußerste verknappt nur das zwischen 1874 und 1915 erschienene Material, eine zum Glück sehr fruchtbare Phase der Rettung heute zum Teil unwiederbringlich verlorener Handschriftenschätze. 
untergehenden alten Europas mit der des großen technologischen Wandels der digitalen Revolution und ihrer elektronischen Vorläufer, die am Ende doch viel einschneidender werden sollte: SMS, Twitter- und Email-Kommunikation. Die digitale Revolution hat aber zwei wesentliche Unterschiede bewirkt: Erstens muss sie schneller gehen, ist knapper und verbindet im Chat sogar Eigentümlichkeiten des Telephons, die den Wechsel der Repliken rascher zusammenführt; zweitens ist das Medium viel volatiler: Kaum jemand käme wohl heute noch auf die Idee, die Speicher eines Anrufbeantworters abzuschreiben statt ihn zu löschen oder die Flut seiner Emails auszudrucken und dann auf Papier aufzubewahren.

\section{Zu Bürgers Briefstil}

\section{Einordnung der stilgeschichtlichen Tradition, Herkunft und Quellen}

Wie alle Gymnasiasten seiner Zeit hatte Bürger im Rahmen des am damaligen Gymnasium üblichen Schreibunterrichts auch das deutsch-lateinische Briefschreiben erlernt. Er löste sich, gleichfalls wie wohl alle seine Altersgenossen alsbald, vielleicht auch schon auf der Schule, davon ab im Sinne der Gellert'schen Briefreform, die m. E. nur den Brief aus der Rhetorik in die Dialektik, die (nicht notwendig eristische) Gesprächslehre überführt hatte. Die dort gelernten Elemente der Unmittelbarkeit (Dialog, historisches Präsens, Unmittelbarkeit des Berichtens) steigerte er freilich zu einem nachgerade szenischen Darstellen.

Stilgeschichtlich steht Bürger damit also in seinen Anfängen noch ganz im Zeichen des empfindsamen Briefs, also dem, was wir heute eigentlich mit dem Privatbrief meinen, in dem der Schreiber bereit ist, seine Seele nach außen zu kehren. Allerdings hat er dem seinen ganz eigenen Stempel aufgedrückt, so dass das stilistische Profil seiner Briefe schier unverwechselbar wurde.

\section{Stilistische Strategien}

\section{Empfängerzugewandtheit}

Dass der Empfänger (gleichviel ob als Imagination oder Konstrukt) nicht bloß den Gegenstand, sondern auch den Stil eines Briefs wesentlich beeinflussen und ihn stärker verändern mögen als der Individualstill eines Autors, ist eine Binsenwahrheit und Eigentümlichkeit des Briefschreibens, Bedingung der Gattung und ihre stilistische Konsequenz. ${ }^{8}$ Nur sehr selbstgefällige oder sehr ungeschickte Briefschreiber passen sich nicht dem gedanklichen und sprachlichen Horizont ihrer Korrespondenten an. Das gebietet nicht nur jede Sozialität und

8. Vgl. Joost, Lichtenberg - Der Briefschreiber, S. 36 f. und passim (u. a. S. 149-151); Albrecht Schöne, Der Briefschreiber Goethe, München, Beck, 2015, hier besonders schlagend demonstriert S. 73 ff. im Kapitel II (An Ernst Wolfgang Behrisch, 10.-13. 11. 1767). 
einfachstes Dekorum, sondern unbedingt auch der gesunde Menschenverstand, sobald es nämlich um den Wunsch nach Ästimation, eine der Haupttriebfedern menschlichen Handelns, und das Interesse am spezifischen movere sich handelt.

Die Briefe Bürgers an Listn zum Beispiel zeigen ein vollkommen anderes sprachliches Profil als den, den wir aus seinen launigen und überschäumend komischen Korrespondenzen mit Dieterich, Scheuffler oder auch Lichtenberg kennen. Die an Boie und Goeckingk sind bei aller Burschikosität vergleichsweise nachgerade keusch und bedienen sich fast nur ,erlaubter Witztechniken wie der Parodie.

Das kann auch ganz indirekt sein: Der wahre Empfänger zum Beispiel jenes berühmten Rechtfertigungsbriefs Bürgers an seine Schwiegermutter über den Ehebruch seiner Frau war ja schon im Augenblick der Abfassung gar nicht diese selbst, sondern ein weiteres Publikum, erst die Richter am Universitätsgericht, die über seine Scheidung und deren Implikationen zu befinden hatten, dann aber die allgemeine Öffentlichkeit, vor der der Dichter zahlreicher Liebesgedichte nun seinerseits sich reinzuwaschen hatte.

Gewiss gilt manches von dem, was ich zu den Briefen an Dieterich gesagt habe, auch für andere Korrespondenzen; die Anverwandlung der lutherbiblischen Sprache zum Beispiel, gleichviel ob in parodistischer oder ernstgemeinter Absicht; das Zitieren von niederdeutschen und schwäbischen Dialektbrocken zur Erzeugung von heiterer Volkstümlichkeit. Dafür tritt gegenüber den Briefen an die Juristen Goeckingk und Listn die wesentlich trockenere juristische Materie und die alltäglichen Sorgen und Geschäftigkeiten hinzu, gleichzeitig aber auch die selbst- und fremdironische Parodie der Rechtssprache.

\section{Reflexion des Abfassungsvorgangs}

Bemerkenswert neu an Bürgers Briefstil ist auch das hohe Maß an Reflexion gegenüber dem Brief selber und der Briefsprache. In meiner Studie zu Lichtenberg hatte ich das mit dem Ausdruck „Metareflexion“, insbesondere „metatextuelle Reflexion" belegen wollen; womit fast exakt das gemeint ist, was die Prager Schule des Strukturalismus (Roman Jakobson, Jan Mukařovský) in Erweiterung der Bühler'schen Sprachfunktionen mit der „phatischen“ Funktion bezeichnet haben. ${ }^{9}$ Am Schluss eines Briefs an Listn, zum Beispiel: „Nun gute Nacht! Es ist heüt fast eben so spät, als da sie Ihren Brief schloßen. Ich geh zu Bette." (an Listn, 23. 2. 1773). Oder „Doch meine Schreibart wird ein wenig alzu körnicht“ (an Listn, 6. 9. 1773).

\section{Elemente der Rhythmik}

Das ist aber nur eine Seite. Bürgers unerhört feines Stilempfinden beweist sich mehr noch als in der Reflektiertheit doch in der Praxis seines Stils, hier also

9. Vgl. dazu sehr eingehend Udo Wargenau, „Über Gottfried August Bürgers Korrespondenz mit Heinrich Christian Boie“, in Lichtenberg-Jahrbuch 2010, S. 153-175; hier besonders S. 167 f. 
dichterisch: immer wieder an der Rhythmik seiner Sprache, auch in seiner Prosa. Es ist augenfällig, dass unter seinen Korrespondenten sich dieses Mittels vor allem sein Förderer und anfangs gewiss auch poetisches Vorbild Gleim in Halberstadt der Technik bedient; spätestens bei ihm hätte er es lernen können (Bürger praktizierte es schon zuvor, hat vielleicht aus Gleims Briefen Anregung und Rechtfertigung bekommen oder hätte sie nachträglich bekommen können). Indes: Bürger behält dieses sichtbare Zeichen eines Formwillens rhythmischer Prosa noch längere Zeit bei - indem er nämlich durch Apostrophen genau markiert, wo in Briefen er Elisionen und Synalöphen gesetzt haben möchte; und das selbst in ganz alltäglichen, ja sogar in eher amtlichen, juristischen Schreiben:

\footnotetext{
„Daher ersuch' ich“ (an die Mitglieder der Deutschen Gesellschaft in Göttingen, 14. 2. 1769) „Und wie konnt' ich?“ (an den Prorektor, 2. 6. 1770)

Ich eilte nach dem letzten Kusse meinem Zimmer zu und kaum kaum bracht' ich meine Augen trocken über die Straße. Mein Herz war mir hoch herangeschwollen, und wären Sie länger geblieben, so hätt' ich mich nicht mehr halten können, so hätt' ich überlaut weinen müssen. (an Gleim, 7. 7. 1771; auch am 29. 9. fünfmal - in dessen Antworten, z. B. vom 12. 8. oder 7. 10. aber sogar dreimal so dicht!)
}

\section{Aposiopese}

Die Aposiopese, das Verstummen, jene rhetorische Abbrechungsfigur, die im 18. Jahrhundert zumeist durch Gedankenstriche, Pünktchen oder Sternchen markiert sind und die nach Lessings Vorgang zu einer Lieblingsfigur des Sturm und Drang-Dramas werden sollte, delegiert gern, wie ich es auch schon bei Lichtenberg exemplarisch demonstrieren konnte ${ }^{10}$, die Entzifferung des Verbotenen und damit den unmoralischen Akt des Aussprechens oder wenigstens Apperzepierens an den Leser - das ist bei Bürger freilich nur höchst selten der Fall. Es sind durchgängig Turpia und Pudenda, die so abkürzt in seinen Briefausgaben erscheinen, aber einige solcher Aposiopesen sind auch erst von den Herausgebern dazu gemacht worden, gar nicht von ihm selber. Denn Bürger hat immer gern alles ausgeschrieben - naturalia non sunt turpia, z. B. (alle an Dieterich); jedoch schriebt er sonst meist die analen und fäkalen Ausdrücke ungescheut aus und ,verstummt " nur bei den sexuellen: „Hosensch-, (23. 10. 1777), „A-,, (10. 4. 1778); „Schw-,, (2. 3. 1778); „Mein Schw... und meine V... empfelen sich Eürem Schw... und eüren V... bestens“ (10. 8. 1780). Man wird also jedesmal erst im textkritischen Apparat nachzulesen haben, wie es sich denn diesmal verhält.

\section{Außersprachlich Ikonisches}

Zeichnungen sind - verglichen etwa mit Lichtenbergs Briefen - bei Bürger selten. Wir haben nur eine (an Dieterich, 1. 1. 1781: ein richtiger kleiner Cartoon) ${ }^{11}$,

10. Joost, Lichtenberg - Der Briefschreiber, S. 226.

11. Fehlt leider noch in meiner Edition des Briefwechsels Bürgers mit Dieterich „Mein scharmantes Geldmännchen“, Göttingen, Wallstein, 1988, weil mir entgangen war, dass Erich Ebstein das 
wissen noch von einer anderen (obszönen), die aber leider verloren ist, an denselben vertrauten Empfänger. ${ }^{12}$

Außerdem gibt es zweimal in seinen Briefen den heiteren Scherz, den ich außer in Bürgers Werk ${ }^{13}$ noch gedruckt bei Matthias Claudius ${ }^{14}$, welchem damit bis auf Weiteres das Erstgeburtsrecht gebührt, und in Briefen Lichtenbergs ${ }^{15}$ gefunden habe: ein Wort auf den Kopf zu stellen, um zu demonstrieren, wie der Schreiber (und der Leser nachher!) den Kopf verdrehen musste, wirklich oder nur moralisch. Der Briefschreiber gibt damit zugleich ein optisches Ironiesignal. In Bürgers Briefen sind es bezeichnenderweise die Wörter „ehrlich“ („einem ehrlichen Manne“: an Bollmann, 25. 3. 1779) - und noch deutlicher „keusch“ (an Dieterich, 28. 5. 1779), das in der Kombination mit „züchtig“ schon beinahe das Ergebnis der Tätigkeit des angeredeten „Verführers des Volks, besonders der Weiber“ Dieterich, von dem da die Rede ist, ikonisch abbildet.

Derlei lustige ikonische Effekte sind aber, selbst wenn wir Bürger unterstellen wollen, dass er sie selber ersonnen hat, weder neu noch einmalig. Aus der Poesie des Spätbarocks, und nicht bloß der heiteren, kenne ich sie, etwa von Johann Christian Günther ${ }^{16}$, und Arno Schmidt, „Wortmetz“ (A. S. über A. S.) des 20. Jahrhunderts, machte in seiner Erzählkunst in den 50er-Jahren des 20. Jahrhunderts sogar ein Programm daraus. ${ }^{17}$

\section{Elemente des, Witzes' (Ingenium) ${ }^{18}$}

Nicht mehr als nur erwähnen kann ich hier, wie schon angedeutet, Bürgers Bildlichkeit, die in ihrer Poetizität und Kühnheit naheliegenderweise seine Zeitgenossen übertrifft, in der Technik der Applikation etwa der compound metaphora aber durchaus nicht an zum Beispiel Lichtenberg heranreicht. Dieser

heute verschollene Original in seiner Ausgabe von Gottfried August Bürger, Des Freyherrn von Münchhausen wunderbare Reisen, o. 0. [Weimar], 1925 (2. Jahresgabe der Bibliophilen), S. 73, schon publiziert hatte.

12. Beilage zum Brief an Dieterich, 1. 11. 1779, die einen Mann zeigte, welcher sich mit der einen Hand an den Hintern fasst, mit der andern seinen langen Penis über ein Gestell legt und die Zunge herausstreckt (eigentlich also einen Priap oder Satyr) mit der Umschrift sapere aude. Original verschollen.

13. Zu Beginn des Münchhausen, wo „höflich“ („einem höflichen deutschen Postmeister“) in der 1. Episode auf den Kopf gestellt ist.

14. In der gedruckten Subskriptionsanzeige vom 20. 8. 1777 (Werke, München, Winkler, 1984, S. 101).

15. Lichtenberg an Wolff, 24. 11. 1783, in Bw 2, Nr. 1194, S. 759 ff.; an Soemmerring, 28. 5. 1784, in Bw 2, Nr. 1270, S. $858 \mathrm{ff}$.

16. In dem Leichencarmen [!] vom 14. 2. 1717: „Billige Thränen über das Absterben der Vorigen [= Cleophe Salome Löscherin geb. Sittigin]. Im Namen ihres Sohnes, beginnt: „Diß ist der Donner-Schlag“, Z. 45 ff. Freundlicher Hinweis von Reiner Bölhoff.

17. Vgl. dazu Ulrich Joost, „Der Au=Tor als d. Säzza, oder: Visuelle, ja audible Etyms? Zu Arno Schmidts Schreibarbeit und Typographiesemiotik“, in Davide Giuriato/Martin Stingelin/ Sandro Zanetti (Hrsg.), „System ohne General“. Schreibszenen im digitalen Zeitalter, München,Wilhelm Fink, 2006 (= Zur Genealogie des Schreibens 3), S. 47-64; hier bes. S. 52.

18. Zur Debatte um diesen Schlüsselbegriff des 18. Jahrhunderts muss ich hier wieder auf Joost, Lichtenberg - Der Briefschreiber, S. 183 u. pass. verweisen. 
Bereich seiner Wortkunst verdiente eine eigene Untersuchung, die die Bildlichkeit in Beziehung zur poetischen Produktion zu rücken hätte.

Selbst wenn man einen dergestalt strengen textlinguistischen Wortspiel begriff wie den durch Christian Wagenknecht am Werk von Karl Kraus entwickelten zugrunde legt, so finden sich einige - wenn auch sehr wenige, so doch köstliche - Beispiele von Wortspielen in Bürgers Briefen; viel weniger übrigens als bei Lichtenberg, aber immer noch bedeutend mehr als etwa bei Lessing oder Georg Forster - von anderen Zeitgenossen gar nicht zu reden. Das Wortspiel ist in jener Zeit auf die rhetorische Paronomasie beschränkt; alle übrigen Typen, wie Shakespeare sie schon theaterfähig gemacht und die Literatur damit bereichert hatte, sind in der deutschen Ästhetik jener Zeit noch verachtet, ja verpönt und haben sich literarische Lizenz erst durch Heinrich Heine, Karl Kraus und (nicht zuletzt) Sigmund Freuds Buch über den „Witz und seine Beziehung zum Unbewussten“ erobert. Ich nenne hier nur als Beispiele „Knabuni“ (an Dieterich, 28. 5. 1779) aus /Rabuni/ (Jesus-Anrede, Luther: ,das heißt Meister') plus /Knabe/; oder, schon etwas blass: „Archilochius“ (an Dieterich, 31. 12. 1792) aus /Archilochos/ (dem griechischen Dichter) plus / Arschloch/ - dieser Witz liegt allzu nahe; ihn bildeten Gymnasiasten bis in meine Schulzeit; ferner „Pöblikum“ (berichtet Althof an Boie, 10. 11. 1794) aus /Publikum/ und /Pöbel/ - „Wollegebohrnes Geschöpf“ (also /Wohlgeborenes/ und /mit Wolle geboren / = Schaf. (An Listn, 15. 5. 1773) und (an denselben, 27. 1. 1773) „Unsere liebe Hausfrau“: aus /Hausfrau/ und /unsere liebe Frau/ (der Bezeichnung für die Gottesmutter Maria).

\section{Einige Quellen Bürger'scher Briefsprache}

\section{Studentensprache}

Studentensprache ist eine wichtige Quelle, burschikos (= studentisch) gab sich Bürger, der sich selber des Pseudonyms Menschenschreck gern bediente, es versteht sich: bramarbasierend. Die Rolle des Bürgerschrecks gefiel ihm überaus gut, wie wir gleich noch sehen werden. Aus der Studentensprache treffen wir daher eine Fülle von Vokabeln, wie Bruder Studio, coram nehmen, Fuchs, Gaudium, Hans Ars (der aus Goethes Faust bekannt sein dürfte), kapores, Knicker, Korydon, Manichäer, Moos (das wie Schmuh ursprünglich rotwelsch ist), pauken, Philister (und Komposita), Pracherei, Pursche, schäkern, Schnakisch, schnelle Kathrine, schnurrig, Schofel, Schubbejackerei, schwul, Schwulitäten, Tausend sa $s a$, wurzeln. Noch kurz vor Bürgers Lebensende sollte Boie noch darüber klagen, dass Bürger seinen burschikosen Tonfall einfach nicht ablegen wolle.

\section{Säkularisations-Elemente}

Das gewiss wirkungsmächtigste Element der Bürger'schen Dichtungs- wie Briefsprache ist fraglos das geistliche Wort: Bibel und Kirchenlied sind die Quellen, 
erworben in einem Pfarrershaushalt, im Gottesdienst und in der Bibelstunde. Er steht selbstredend nicht allein da, denn die biblische, überhaupt die christlich religiöse Sprache, also auch aus Gesangbuch und Erbauungsliteratur, sind selbstverständlicher Bestand einer christlichen Erziehung und damit allseits verfügbarer Bildungshorizont. ${ }^{19}$

Allein im ersten Band unserer Edition habe ich gegen 230 Bibelanspielungen gezählt! (und wer sagt, dass ich alle bemerkt habe!). Dazu kommen noch 15 Anspielungen auf protestantische Kirchenlieder. Derlei Allusionen gehorchen in ihrer Verwendung den strukturellen Regeln der sprichwörtlichen Redensart, zum Beispiel: „ob sie denn wircklich da säßen wo Heülen und Zähneklappen“ (an Listn, 15. 4. 1773). ${ }^{20}$

Freilich war dieses Diktum aus dem Evangelium selber schon und auch zu Bürgers Zeit sprichwörtliche Redensart, ja war das wahrscheinlich sogar schon in der Bibel: Allein bei Matthäus begegnet die Wendung sechsmal. Aber unser Briefschreiber lehnt sich eben eng an Luthers Wortlaut an, gibt mithin Wiedererkennungssignale, die den blasphemischen Charakter von solcherart Zitieren sinnfälligwerden lässt. Es würde bei Bürger nachgerade überraschen, wenn nicht in den meisten Fällen seine biblischen Anspielungen und Zitate anders daherkämen denn als weltliche Kontrafakturen oder schlichtweg Parodien, wie uns ja schon vor mehr als einem halben Jahrhundert Albrecht Schöne belehrt hat. Es sei hier nur an einem einzigen, wiewohl hinreißenden Beispiel demonstriert, wie Bürger eine wilde Kombination von insgesamt über 30 Bibelstellen bietet, scheinbar ganz wertneutral dicht am Biblischen, oder wie sich Steinhausen ${ }^{21}$ noch ausdrückt, „im Tone des alten Testaments“ (freilich nicht nur, das Neue Testament kommt fast ebenso oft vor). Diese Kollage transportiert eigentlich nur die recht banale Information, dass der Empfänger in Göttingen eine alte Schuld für ihn eintreiben und damit seine Reise in seine Heimatstadt Lübeck finanzieren soll (An Johann Matthäus Tesdorpf, 6. 5. 1773) - deswegen also steht sie nicht da, sondern um des Spaßes willen, der mit der Heiligen Schrift kontrafaktorisch getrieben wird, und der Artifizialität der Kombination wegen (beim folgenden Zitat mussten ausnahmsweise die Anmerkungen der neuen Briefausgabe zum Nachweis der Stellen alle mitgeführt werden):

Geh hin ${ }^{1}$ gen Wehrs an den FleischScharn ${ }^{2}$ und Siehe! ${ }^{3}$ da wirst Du angebunden finden Sechs Louisd'or, löse sie und führe sie zu mir. Und so Dich Jemand fragen wird, so sprich: Der Herr bedarf ihrer, und alsbald wird er sie Dir laßen. ${ }^{4}-$

Denn Siehe! Du Menschenkind, ${ }^{5}$ Farren $^{6}$ haben mich umgeben und fette Ochsen halten mich umringet; und ich befahre ${ }^{7}$, daß der Widersacher ${ }^{8}$ mich übergebe dem Richter, und der Richter überantworte mich dem Peiniger ${ }^{9}$ und ich werde geworfen in den Kerker, alwo

19. Man vergleiche nur das 120 Seiten starke Bibelstellenregister, das Günther Arnold im 17. Band seiner Edition der Herder-Briefe (Weimar, Böhlau, 2014, S. 109-226) beigefügt hat. Das Verzeichnis der Sprichwörter hingegen braucht nur 35 Seiten (S. 227-261)!

20. Strodtmanns Edition verballhornt zu „Zähneklappern“; von Dispeker in einer zuverlässigen Abschrift des Originals korrigiert. Diese Fassung ist dichter an Luthers originaler, schon im 19. Jahrhundert modernisierter Bibelübersetzung!

21. Georg Steinhausen, Geschichte des deutschen Briefs. Zur Kulturgeschichte des deutschen Volkes, Bd. 2, Berlin, Gaertner, 1891, S. 294. 
wird seyn Heülen und Zähnklappen, ${ }^{10}$ von dannen ${ }^{11}$ ich nicht wieder heraus komme, bis ich den letzten Heller ${ }^{12}$ bezahle. | Das Wort des Herrn geschah ${ }^{13}$ zu Bürger dem Sohn Gertraut, der Tochter Bauers als er lag Nachts auf seinem Bette, und sprach: Du Menschenkind, ich will fegen Deinen Beütel, wie man eine Tenne feget, ${ }^{14}$ und es soll derselbe leer seyn, wie eine Wasserblase ${ }^{15}$, und soll derselbe liegen auf dem Söller ${ }^{16}$ unter der Banck ${ }^{17}$ und sollen darinn die Spinnen ihr Gewebe aufziehen und darinn herbergen allerley Gewürm das auf Erden kreücht. ${ }^{18}$

Und Du Menschenkind, ich will Dir erwecken einen Widersacher ${ }^{19}$ von Abend, des Nahme genennet wird Habernickel ${ }^{20}$, und er soll gegen Dich ausziehn und mit den Reisigen ${ }^{21}$ des Processes und Dich ängstigen viele Tage lang. ${ }^{22}$ | Und Du Menschenkind, wirst vielen Jammer haben, und wirst schreyen nach Gelde, wie der Hirsch schreyet nach frischen Wasser. ${ }^{23}$ Und zu der Zeit werden wenige kommen und Recht von Dir kaufen. Denn Siehe! der Lenz ist kommen ${ }^{24}$ und die Baüme haben Knoten gewonnen und der Sämann ist ausgegangen zu säen ${ }^{25}$ und kann nicht warten des Haders vor dem Gerichte. ${ }^{26}$

Und Du Menschenkind, zu dieser Zeit will ich Dir bescheeren dreyßig Silberlinge ${ }^{27}$ und davon sollst Du leben in der theüren Zeit; und ich will für Dich das weitere ersehen nach diesen; und will Dich führen gen Mitternacht ${ }^{28}$ an das Meer in die Stadt, so da heißet ${ }^{29}$ Lübeck, | und will Dir geben zum Weibe ${ }^{30}$ die reiche Tochter eines Crämers; und Du wirst empfahen ${ }^{31}$ viel Silber und Gold und Knechte und Mägde und wirst reich und mächtig werden vor Deinem Volck. ${ }^{32}$ Und Du wirst zeügen Söhne und Töchter, ${ }^{33}$ welche sollen auffliegen wie Adler zu der Sonnen ${ }^{34}$ und will ihnen geben lieblichen Gesang in ihre Kehlen, und die Kunst die Harfe zu spielen, ${ }^{35}$ gleichwie ich Dir gegeben habe. Und Du wirst viel Freüde haben bis Du versammlet werdest zu Deinen Vätern. ${ }^{36}$

Und das Wort des Herrn geschah abermal zu mir ${ }^{37}$ und sprach: Du Menschen kind, schreib auf dieses Gesicht ${ }^{38}$ und sende es gen Göttingen an Tesdorpf, aus der Stadt Lübeck so da lieget am Meer; und ich thät gleichwie der Mund des Herrn geboten hatte. ${ }^{39}$

[Schnörkel]

Allerdings: Der Brief parodiert für damalige Verhältnisse bis an die Grenze des Blasphemischen oder über diese hinaus (was den Schnörkel an seinem Schluss, der die Unterschrift ersetzt, erklären würde!) die Luther'sche Bibelsprache sowohl durch intensiven Gebrauch von seinerzeit bereits veralteten Konstruktionen (z. B. Partizip ,kommen' [= gekommen], warten' mit Genetiv statt mit Präposition; relativischem Anschluss mit Correlativum ,so statt mit Pronomen; Nachbildung der für Luther typischen Satzstellung mit vorgezogenem Prädikat, z. B. ,will Dir geben zum Weibe', ,wirst zeügen Söhne und Töchter oder durch nur mehr dichtungssprachlich verwendete Wörter und Wendungen (z. B. ,von dannen'[= von wo], gen' [= hin, auf ... zu, gegen]), poetische Umschreibungen wie die Himmelsrichtungen durch die Tageszeiten (,Abend', ,Mitternacht‘ für Westen bzw. Norden) als auch und vor allem durch zitierende Anspielungen. Diese über 30 alludierten Bibelstellen sind nachstehend im Einzelnen nachgewiesen. ${ }^{22}$

- 1 vgl. z. B. Mt 8, 9; hier v. a., wie aus dem Folgenden erhellt, Mt 21, 2.

- 2 auch ,Fleischbank': der Ort einer Stadt, wo die Schlachter ihre Ware öffentl. feilbieten; sie befanden sich in Göttingen damals an der Nordostecke der Johanniskirche zum Rathaus und Markt gerichtet, eben dort, wo Wehrs wohnte. Tesdorpf logierte damals im Hause des Publizistik-Professors August Ludwig Schlözer an der Ecke Gotmar- und Paulinerstraße, also nicht weit entfernt.

- 3 vgl. Mt 21, 5 (u. ö.!), das Folg. wieder nach Mt 21, 2.

- 4 vgl. Mt 21, 3.

22. Zu Bürgers produktiver Umsetzung des im geistlichen Elternhause aus Bibel und Kirchenlied Gelernten vgl. A. Schöne, „Weltliche Kontrafaktur. Über Bürgers Lenore“, in ders., Säkularisation als sprachbildende Kraft. Studien zur Dichtung deutscher Pfarrersöhne, 2., überarbeitete und ergänzte Aufl., Göttingen, Vandenhoeck \& Ruprecht, 1968, S. 181-224. 
- 5 vgl. Hes 2, 1; - Nr. 128.

- 6 vgl. 1. Mos 32, 15; - Nr. 112.

- 7 befürchte.

- 8 vgl. Hebr 10, 27.

- 9 vgl. Mt 18, 34.

- $10 \mathrm{vgl}$. Mt 8, 12.

- 11 ,von wo“; kommt über 100mal in Luthers Übersetzung vor; in Verbindung mit dem Folgenden aber eindeutig bezogen auf Lk 12. Mt 5, 26.

- 12 vgl. Mt 5, 26.

- 13 vgl. Jer 1, 4 u. Jer 7, 1.

- 14 vgl. Mt 3, 12; zu Tenne - Nr. 72.

- 15 Schaum-, später auch Seifenblase, spätestens seit dem 17. Jh. (Andreas Gryphius: Eitelkeit menschlichen Lebens) und bis ins späte 18. Jh. (Christoph Hölty!) poetisch als Vergänglichkeitstopos viel gebraucht und vielleicht aus der Emblematik (Homo Bulla) übernommen als Sinnbild für nutzloses Tun, unbeständige Liebesgunst und leere Gerüchte (vgl. A. Henkel / A. Schöne: Emblemata 1967, 1315-1317). Begegnet bei Luther zwar auch öfter (Grimms DWb gibt Belege aus der WA Luthers Bd 7, 586. 10/II, 55. 16, 434. 33, 629), aber anscheinend nicht in der Bibelübersetzung.

- 16 in der hier gemeinten Bedeutung ,Fußboden“ begegnet der Ausdruck in der Apg 98, 37.

- 17 unter die Bank Lk 8, 16; aber auch sonst häufig in Luthers deutschen Schriften für ,unterdrücken', ,wegwerfen', ,übergehen', ,verstecken' (und so noch von Thomas Mann in seinem Doktor Faustus pasticheartig gebraucht!).

- $18 \mathrm{vgl}$. 1. Mos 1, 26.

- 19 in der Bibel oft, aber nicht immer, der Teufel, das Prinzip des Bösen.

- 20 über den auch von Lichtenberg sehr geschätzten Dr. jur. u. Göttinger Anwalt, der demnach seinerzeit B.s Gläubiger zu vertreten hatte, vgl. L.s Bw 1 u. 5/1. Er kam tatsächlich von Abend (= Westen, wie es an zahllosen Stellen der Lutherbibel heißt), stammte nämlich aus Gimborn in Westfalen.

- 21 reitende Knechte, Soldat zu Pferde (Adelungs GKW). In der Bibel in 1. Mos 50, 9 u. a.

- $22 \mathrm{vgl}$. Ps 13, 3.

- 23 vgl. Ps 42, 2.

- $24 \mathrm{vgl}$. Hohelied 2, 12.

- $25 \mathrm{vgl}$. Mt 13, 3.

- 26 ,kann nicht auf den Streit warten“ - anscheinend so nicht lutherbiblisch. Vgl. aber Sie sollen uns nicht für ihre Gerichte und Hadermärkte ziehen (Luther's Tischreden, 191a Saltzmann, Anh. 38a, zit. in W. Wander: DSL 2, 1870, 249), und Wenn ein Hader ist zwischen Männern, so soll man sie vor Gericht bringen (5. Mos 25, 1.).

- $27 \mathrm{vgl}$. Mt 26, 15.

- 28 nach Norden; vgl. z. B. Hes 8, 14 (bei Luther dort aber: gegen Mitternacht!).

- 29 ähnliche Konstruktion z. B. Lk 9, 10.

- 30 vgl. z. B. 1. Mos 29, 28.

- 31 schon damals veraltet für, empfangen: In der Bibel z. B. 5. Mos 9, 9.

- 32 anscheinend nicht lutherbiblisch, jedoch begegnet die Wendung, die demnach seinerzeit ganz kommun gewesen sein muss, in der Vossischen Ilias-Übersetzung 1, 91.

- 33 vgl. Jer 29,6.

- $34 \mathrm{vgl}$. Jes 40, 31.

- 35 anscheinend nicht lutherbiblisch; vgl. aber B.s Danklied (Nr. 72, GMA 1773, 193): Vor Tausenden gab deine Gunst | Des Liedes und der Harfe Kunst | In meine Kehle, meine Hand; | Und nicht zur Schande für mein Land!

- 36 vgl. 1. Mos 25, 8.

- 37 vgl. 1. Mos 35, 9.

- 38 Vision; vgl. Hab 2, 2. - auch Nr. 115 (NachtGesicht).

- $39 \mathrm{vgl}$. Jes 58, 14.

Die Beispiele aus dem Bereich der sakralen Sprache ließen sich fast beliebig vermehren: es ist jedenfalls die Textgruppe mit dem höchsten Anteil bei Bürgers Intertextualitäten. 
Oft ganz belanglose oder zumindest alltägliche Sachverhalte erscheinen so im Gewande von Luthers Sprache, freilich mit überraschenden Wendungen und Kombinationen, die man bei dem frommen Stoff nicht gleich erwartet hätte, und nicht im direkten Zitat, sondern durch Nachahmung des archaischen Ethos („,ür“ statt „Vor“) und Vokabulars („Lenden“ - in Luthers Sprache waren das eben die Keimdrüsen, und das wusste noch jeder Zeitgenosse Bürgers) ${ }^{23}$ :

An die FilzPantoffeln bin ich, wie die Jungfer zum Kinde gekommen. Wir machten das Packet auf und klopften für Freüden die Lenden, als wir die stattlichen Pantoffeln erblickten. (An Listn, 15. 3. 1773)

Überreich sind die Briefe insbesondere der frühen Jahre an emotionalen Vokabeln jener Freundschaftskultur. Wir wissen heute, wie sehr der Freundschaftston der Empfindsamkeit sich einem säkularisierten Pietismus verdankt; so auch bei Bürger, freilich wohl selten ganz direkt: Im Stammbuchblatt, in den frühen Briefen an die Mentoren Klotz und Gleim begegnet natürlich gehäuft die Lieblings- und Reizvokabel der Epoche: „Herz“ - sie hat dort schon den religiösen Hintergrund des cor Christi vergessen. Aber bei Kompositabildungen wie der Anrede „Herzensboie“ (z. B. an Boie, 10. 5. 1773; 5. 12. 1776) schimmert die einst von Shimbo herausgearbeitete Übertragung der Liebe zu Gott auf den Mitmenschen noch sehr deutlich durch. ${ }^{24}$

\section{Elemente des Mündlichen und der Umgangssprache}

Zum ästhetischen Konzept von Volkstümlichkeit gehört, auch zugleich passend zur „Schönen Natürlichkeit“ des Briefschreibens, die Verwendung von sonst durchaus subliterarischen und daher eigentlich durchaus nicht gern gesehenen Elementen mündlicher Rede wie exclamatio und Onomatopoie (Ausruf und Lautmalerei). Ich muss mich hier wieder mit der bloßen Auswahlaufzählung mit den Nachweisen und wenigen ergänzenden Hinweisen zufrieden geben:

„Anschnarchung“ (28. 10. 1776 an Boie); „Knarren“ (an Boie, 11. 4. 1776); , mit den Zähnen knirschen“ (an Listn, 29. 3. 1773 und öfter); „nökern“ (1. 11. 1779 an Dieterich); „Kikelkakel“ (5. 12. 1776) bzw. „Kik Kak“ (an Klotz, 19. 2. 1769); „Schnickschnack“ (an Boie, 8. 7. 1773); „Lirumlarum“ (an Boie, 10. 7. 1775) entstammt dem Kinderspiel und Kinderlied. Den häufigen Gebrauch von „Hurrah“ und „Hurre“ in den Briefen an Boie (z. B. 9. 9. und 16. 9. 1773; 31. 7. 1775) oder Gleim (2. 1. 1776) teilt er aber mit den Korrespondenten. Immerhin: Die berühmte Zeile aus der Lenore („Hurre, hurre hop hop hop“) ist vermutlich der bekannteste und meistzitierte Vers Bürgers bis ins beginnende 20. Jahrhundert gewesen. Rätselhaft ist mir bislang noch das „Enkühhhh“ aus der Korrespondenz mit Goeckingk (2. und 13. 10. 1776).

23. Sie begegnen am 5. April 1773 nochmals - natürlich wieder mit der sexuellen Konnotation.

24. Sukeyoshi Shimbo: „Die innerpietistische Säkularisation des Bekenntnisbriefes“, in DVjs Bd. 56, 1982, S. 198-224. 


\section{Fremde Zungen: Der Dialekt in Bürgers Briefen}

Ungefähr dreißigmal fällt Bürger im ersten Band der neuen Ausgabe ins Plattdeutsche seiner Umgebung. Dass er dieses Idiom im protestantischen Pfarrhaus seiner Kindheit aktiv gelernt hat, darf man füglich bezweifeln: Der niederdeutsche Raum ist zwar bis zum Ersten Weltkrieg wenigstens auf dem Lande und in kleineren Städten vollständig, bis zum Ende des 19. Jahrhunderts selbst in größeren zumindest teilweise zweisprachig. Aber Bürger war das nach genau der Maßgabe, die ihn vermutlich davon getrennt hat, nur passiv: In der Kirche und auf dem Rathause wurde nämlich Hochdeutsch gesprochen. Bürger verstand diese Mundart also mit Bestimmtheit, übersetzte auch, wie die Gerichtsprotokolle zeigen, die Aussagen seiner Bauern ins Hochdeutsche, aber es ist nicht seine Mutter- und Erstsprache gewesen. Nur fügte sich das Plattdeutsche großartig in seine Konzeption einer Erneuerung der Dichtung aus der Volkssprache. Und so streut er regelmäßig einzelne niederdeutsche Wörter oder Wendungen, sehr selten auch einen ganzen Satz in seine Briefe ein. Am ehesten echt also klingt noch das Plattdeutsche, zum Beispiel (alle an Listn) „Wir wollen alsdenn leben, as de Pewinzen“ (23. 2. 1773); oder „Use Frue“ (16. 9. 1773); oder „wenn he man sine annern Saaken träft, dit sall je wol weren“ (21. 6. 1773). ${ }^{25}$

Etwas albern, zumal da Bürger mit dem oberdeutschen Dialekt gar nicht zurechtkommt, wirken seine Versuche im Schwäbischen ${ }^{26}$

Ey! wolscht auf Maschkeraaten gehn? ${ }^{27}$ Wärscht dabor heüt hier gewesche, und hättscht die Maschkeraat gesehen, die der Göttingische Satan wieder angestellt hatte. (An Listn, 23. 2. 1773)

ähnlich an denselben Empfänger, 15. 4. 1773:

Es ist uns herzlich lieb, daß Sie so fromm werden. Scho? Kannscht nun fromm werde? Kannscht in die Kirche gehen? Kannscht andächtige Briefe schreibe? bischt mürbe geworden? hättscht schon lang dasch Ungersch hochadliche Weschen ablegen können, und statt auf Maschkeraten in die Kirche gehen können.

\section{Volkstümliches und Volksabergläubisches}

Allein im 1. Band finde ich an die 80 sprichwörtliche Redensarten und Anspielungen auf Volkssitten. Manches davon hat Bürger gar nicht selber „im Volke“ aufgeschnappt. Solche Redensarten sind eben nicht dem Volke abgelauscht, sondern waren wahrscheinlich, wie lexikalisch verbürgtes Alter oder Landschaft andeuten, vermutlich in Büchern aufgelesen bei solchen

25. Wenn er nur seine andern Sachen träfe, dann soll es schon werden.

26. Steinhausen, Geschichte des deutschen Briefs, S. 279 stellt den Dialekt im Brief richtig ins Kapitel „Natürlichkeit und Freiheit“, hält ihn aber offenbar für ein reines Sturm- und-Drang-Element was er ganz gewiss nicht ist, auch bei Lichtenberg begegnet er, wiewohl nicht mit der dramatisch offensiven Attitude, auch.

27. Eine (nicht besonders gute) Nachahmung von schwäbischer Mundart. 
Autoren wie dem bewunderten Dichter Johann Christian Günther. Aber vieles könnte er doch tatsächlich von seinen ländlichen Umwohnern gelernt haben. Erstaunlich viel der sprichwörtlichen Redensarten ist noch heute kurrent oder zumindest verständlich, alles aber trägt zum Eindruck der Lebendigkeit seines Stils bei. Um nur ein paar Beispiele zu nennen: Er ,spricht frisch von der Leber weg“ (an Listn, 1. 2. 1773), ,sitzt in den Rosen ' (an Boie, 18. 5. 1772),verkauft um ein Butterbrot'(an Listn, 29. 3. 1773); er ,leckt den Bären' (an Goeckingk, 29. 1. und 29. 4. 1776; = bringt etwas in Form); findet: „das dank euch Herodes [= der Teufel]“ (an Boie, 22. 4. 1773) oder will sich einen „Parra, d. i. der LeichenVogel der Römer“ anschaffen (an Boie, 14. 8. 1773) - das geht bis hin zu der Anrede: „du Auflecker seiner PomeranzenExcremente“ (an Cramer, [Mitte November 1773]), eine Anspielung auf die sprichwörtliche Redensart ,einen prügeln, dass er Pomeranzen scheißt: ${ }^{28}$ (wollen wir hoffen, dass er sie nicht bei den Pietisten in Halle gelernt hat). Und dieses letzte Beispiel führt mich unweigerlich zu dem nicht häufigsten Phänomen, welches aber ganz gewiss Bürgers Stil in der öffentlichen Wahrnehmung am stärksten geprägt hätte, wäre in früheren Editionen nicht so viel davon unterdrückt worden:

\title{
Grobianisches
}

Einige der hier dargestellten Stilmerkmale und -strategien finde ich wenigstens ansatzweise natürlich auch bei anderen Briefschreibern seiner Zeit, etwa bei Lichtenberg oder Georg Forster. Aber nirgendwo wird die Physiognomie des Briefstils so vordergründig wie nachhaltig geprägt durch vor allem zwei Elemente wie bei Bürger; durch die schon dargelegte Säkularisation nämlich und durch seinen Grobianismus. Beides sind, wie ein Blick in die Briefe des jungen Goethe lehrt, auch Berührungspunkte mit dessen Briefstil. Der Grobianismus steht zudem in einer im Deutschen höchst wechselvollen, aber nie ganz durch ästhetische Begrenzungen und Verbote unterdrückten Tradition. ${ }^{29}$

An Dieterich meldet er am 2. 3. 1778:

\begin{abstract}
„Das ist mir mal wieder ein rares Stückchen Brief! Um Gotteswillen! lieber Dietrich, ihr last doch wohl Eüre Leüte im Laden meine Briefe nicht aufbrechen? Nun wahrhaftig! die würden mich für einen artigen Schweinepelz halten. Um des Himmelswillen! zerreist sie gleich, wenn Ihr sie gelesen habet. Ich werde künftig keinen Nahmen mehr drunterschreiben, oder mich allenfals Hosius Pomposius nennen. Daß Ihr sie Eüre Töchter nicht lesen last, dafür kann ich wohl sicher seyn. Eüre Christel aber kann sie wohl lesen; denn die darf schon ein Wörtchen mitsprechen.
\end{abstract}

Die Grobianismen Bürgers sind ganz überwiegend sexuell oder anal beziehungsweise fäkal. Im Sachregister zum Bürger-Dieterich-Briefwechsel, in dem dieser Bereich auch die höchste Dichte hat, ist das einschlägige Vokabular einigermaßen vollständig ausgeworfen und mithin zählbar; es begegnen da also in knapp 100 Briefen „Arsch“ und Komposita: 15mal, „hofieren“ (= seine 
Notdurft - auf dem Hof - verrichten): 3mal, „scheißen“ und Komposita (also etwa auch „Hosenscheißer“): 35mal, „Votzen“: 2mal, „Schwänze“: 5mal, „furzen“: 2mal. Dazu treten eine Reihe von weniger derben - übrigens dadurch witzigeren Variationen wie „Hosius Pomposius“, „Hosentrompeter“, „Archilochius“ (also Wortspiel, s. o.): Wer's nicht glaubt, sehe selbst nach.

Hier noch ein paar unbekannte oder weniger beachtete Spitzenleistungen zur Erbauung. Man glaube bitte nicht (ich halte es zumindest für abwegig), dass diese eruptiven Entladungen einer zugegeben ziemlich juvenilen Phantasie mehr sind als Bürgerschreck und Provokation und etwas zu tun haben mit spezifischen Neigungen, wie sie in den sexual-anthropologischen Schriften des Samuel Krauss oder den Anzeigen gewisser heutiger Zeitschriften breitgetreten werden:

Mein unbesonnener Streich, hatte mir zwar auch einige Unruhe, schon vor Erhaltung ihres Briefes gemacht, aber so fürchterlich ist er mir nicht vorgekommen, als Ihnen. Nunmehro ist mir freylich etwas schissern ${ }^{30} \mathrm{zu}$ Muthe; allein wir großen Geister, wenn wir in den Pott gesch.. haben, so fressen wir es so gut wieder aus als wir können. (an Listn, 18. 2. 1773)

Und schließlich an Lichtenberg: Das fängt noch vergleichsweise harmlos an, studentische Kritik am ,Philister, am Kleinbürger, mischt sich mit Bibelsprache:

Wie es die ehrlichen Philister machen, daß sie so fein ordentlich und regelmäßig einen Tag wie alle Tage leben, jedes Ding, als essen, trinken, schlafen, arbeiten, ihre Weiber erkennen u. s. w. so hübsch nach Salomons Vorschrift zu seiner Zeit thun, das mogte ich warlich wol wissen. (An Lichtenberg, 8. 1. 1780)

Aber dann lässt er sich von seiner eigenen Suade hinreißen, steigert sich ins Grobianische; dergestalt, dass 80 Jahre nach seiner Wiederauffindung niemand gewagt hatte, diesen Brief zu publizieren, ${ }^{31}$ und sein Original sogar verschollen, vielleicht vernichtet ist. Man könnte bei der Lektüre mancher seiner Briefe (und so besonders auch bei diesem hier) auf die Idee kommen, er habe am Tourette-Syndrom gelitten. Das hätte einen Tiefenpsychologen wie Freud (an-dessen Deutungskompetenz bei allem Anregungscharakter ich nicht glaube) mit Freude oder einen Joyce mit joy erfüllt, wie Arno Schmidt einmal witzelte.

30. Vgl. die Lieblingsvokabel des jungen Goethe „scheißig“; vornehmlich gebraucht im Verkehr mit Johann Heinrich Merck; so etwa „scheissig gestrandet“ (im Erstdruck zu „garstig“ verfälscht) an Merck, zwischen 13. und 25. August 1775 (J. H. Merck, Briefwechsel, hrsg. von Ulrike Leuschner in Verbindung mit Julia Bohnengel/Yvonne Hoffmann /Amélie Krebs, Bd. 1, Göttingen, Wallstein, 2007, S. 580 = Goethe, Briefe. Historisch-kritische Ausgabe, hrsg. von Georg Kurscheidt und Elke Richter, Bd. 2,1, Berlin, Akademie-Verlag, 2009, S. 207 = WA IV 2, 1887, S. 278, Nr. 345); wiederum auf sein neues Amt bezogen („das durch aus scheisige dieser zeitlichen Herrlichkeit“) an denselben, 22. Januar 1776 (Merck, Briefwechsel, Bd. 1, Nr. 190, S. 615 = WA IV 3, 1888, S. 21, Nr. 345; auch in: Briefen an Merck, hrsg. von Karl Wagner, Darmstadt, Diehl, 1835, S. 120, 69, 59 bzw. Schriften und Briefwechsel, hrsg. von Kurt Wolff, Bd. 2, Leipzig, Insel, 1909, S. 74); ferner an Lavater, 4. März 1777: „Scheisgesicht“ (WA IV 3, 1888, S. 138, Nr. 565). Und damit wird es schwerlich sein Bewenden haben.

31. Bis ich ihn aus einer zuverlässigen, wiewohl schwer zu entziffernden Abschrift um 1900 in den 2. Bd. der Edition von Lichtenbergs Briefwechsel (1985) aufnehmen konnte. 